\title{
Sensitivity and specificity of 24-hour urine chemistry levels for detecting elevated calcium oxalate and calcium phosphate supersaturation
}

\author{
M. Adrian Rossi, MD; ${ }^{*}$ Eric A. Singer, MD; ${ }^{*}$ Dragan J. Golijanin, MD; ${ }^{*}$ Rebeca D. Monk, MD; ${ }^{\dagger}$ Erdal Erturk, MD; \\ David A. Bushinsky, MD ${ }^{\dagger}$
}

See related article on page 123

\begin{abstract}
Objectives: The gold standard for determining likelihood of calcium oxalate $(\mathrm{CaOx})$ and calcium phosphate (CaPhos) stone formation in urine is supersaturation of $\mathrm{CaOx}$ and $\mathrm{CaPhos}$. Our objective was to investigate whether traditional measurement of total calcium, oxalate and phosphate in a 24-hour urine collection is sufficiently sensitive and specific for detecting elevated supersaturation to preclude the more expensive supersaturation test.
\end{abstract}

Methods: We performed a retrospective review of 150 consecutive patients with nephrolithiasis who underwent measurement of $\mathrm{CaOx}$ supersaturation ( $\mathrm{CaOxSS}$ ) and CaPhos supersaturation (CaPhosSS), as well as total calcium, oxalate and phosphate in a 24-hour urine collection. We used various cut-off values to determine sensitivity and specificity of 24-hour urine measurements for detecting elevated $\mathrm{CaOxSS}$ and CaPhosSS.

Results: In men and women, the sensitivity of 24-hour calcium for detecting elevated $\mathrm{CaOxSS}$ was $71 \%$ and $79 \%$, respectively; for oxalate, sensitivity was $59 \%$ and $36 \%$, respectively. In men and women, the sensitivity of 24 -hour calcium for detecting elevated CaPhosSS was $74 \%$ and $88 \%$, respectively; for phosphate, sensitivity was $57 \%$ and $8 \%$, respectively. In men and women, the specificity of 24-hour calcium for detecting elevated CaOxSS was 55\% and 48\%, respectively; it was $60 \%$ for detecting elevated CaPhosSS in both men and women.

Conclusion: Traditional 24-hour urine analysis is sensitive, but not specific, for detecting elevated CaOxSS and CaPhosSS. Most patients with abnormal 24hour urine analysis have normal supersaturation, and treatment decisions based on traditional urine analysis would lead to overtreatment in these patients.

CUAJ 2008;2(2):117-22

\section{Introduction}

There are several physiochemical features important for stone formation, including supersaturation, nucleation, crystal growth and aggregation. The thermodynamic driving force for stone formation, though, is supersaturation. ${ }^{1}$ As the supersaturation of a substance in the urine increases, so does the size and the number of crystals formed, as was recently demonstrated in an in vitro model of calcium oxalate (CaOx) crystal morphology. ${ }^{2}$ Urine supersaturation in patients with nephrolithiasis is well correlated with the crystalline component of stones passed by those patients, both in a university setting and in a private practice setting. ${ }^{3,4}$ Shipping samples to a central laboratory does not alter measurement of supersaturation for $\mathrm{CaOx}$, calcium phosphate (CaPhos) or urate supersaturation. ${ }^{4}$

Evidence suggests that urine supersaturation can be used to follow treatment response in patients with stone disease. When patients with elevated supersaturation are treated, there is a proportional reduction in the level of supersaturation. ${ }^{5}$ This was confirmed by Siener and colleagues ${ }^{6}$ in a prospective study of known $\mathrm{CaOx}$ stone formers who were medically treated. Patients who remained free of stone recurrence exhibited a significant decrease in $\mathrm{CaO}$ x supersaturation ( $\mathrm{CaOxSS}$ ), whereas those in whom stones recurred had a statistically significant elevation of $\mathrm{CaOxSS}$.

For most laboratories, supersaturation determination requires sending a urine sample to a central laboratory and is likely more expensive than measuring total 24-hour urine levels in-house. ${ }^{7}$ Because of the added expense and the shipping requirements, the supersaturation test may not be as readily available in all locations as traditional 24-hour urine chemistry analysis. The goal of our study was to test the hypothesis that traditional 24-hour urine levels of calcium, oxalate and phosphate are sufficiently sensitive and specific to preclude the need for urine supersaturation testing. 
Although urinary $\mathrm{pH}$ is a critical factor in stone formation, the measurement of $\mathrm{pH}$ by $\mathrm{pH}$ metre tends to be done only by laboratories that report urine supersaturation and therefore does not address the question of whether using referral laboratories is beneficial. Since most patients in our population form calcium-containing stones, we focused on $\mathrm{CaOxSS}$ and $\mathrm{CaPhos}$ supersaturation (CaPhosSS).

\section{Methods}

\section{Patients}

A retrospective chart review was performed on 150 consecutive adult patients (84 men, 66 women) with known nephrolithiasis who underwent testing for urine supersaturation of $\mathrm{CaOx}$ and CaPhos. Included with the supersaturation test was simultaneous measurement of 24-hour urine calcium, oxalate and phosphate levels. Samples were collected with patients on their regular diets. Urine samples were sent by express courier to Litholink Corporation (Chicago, III.), where the tests were performed. CaOxSS and CaPhosSS were calculated with EQUIL2. ${ }^{1}$ This calculation represents the supersaturation ratio, which is the activity product (of calcium and oxalate ions, for instance) divided by the solubility product. ${ }^{8}$

\section{Cut-off values}

Normal values for traditional 24-hour calcium, oxalate and phosphate were based on sex-specific ranges used by the laboratory at our institution and are consistent with established definitions of normal levels. For CaOxSS and CaPhosSS, cut-offs used by Litholink Corp were used. These cut-offs are based on previous studies ${ }^{3,4}$ and are more sensitive.

\section{Stone analysis}

Stones were analyzed by commercial laboratories chosen by the patient's physician. Presence of a phase was defined as any value above zero, and absence defined as none in any stone. Stones were categorized as calcium-containing, uric acid stones (without calcium component) and "other." If a patient had more than 1 stone analyzed, the 2 most recent were included. No patient had more than 2 stones analyzed.

\section{Treatment}

Patients were considered to be on medical treatment if they were taking a drug or were on a diet aimed at improving metabolic abnormalities in stone disease. These treatments included a thiazide, citrate, allopurinol, alkalinization, amiloride or magnesium oxide. The prescription of specific drugs was at the discretion of a urologist or nephrologist. Dietary treatment consisted of counselling by a physician about changes that can decrease stone formation. Specifically, patients were instructed to drink at least $2 \mathrm{~L}$ of water daily, limit sodium, limit protein intake (45 g for women, $60 \mathrm{~g}$ for men) and follow the Recommended Daily Allowance for daily calcium intake. Patients were given a handout with these instructions as well as a list of specific foods to avoid. The types of previous surgical treatment for calculus disease in this population were also explored. All surgical interventions performed to remove a stone or help with its passage were counted and included shock wave lithotripsy, ureteroscopic stone manipulation, ureteroscopic lithotripsy, stent placement and percutaneous and open procedures.

\section{Sensitivity}

For the sensitivity analysis, true positives (TP) were defined as patients who had elevated 24-hour urine levels (of calcium, oxalate or phosphate) and also had elevated $\mathrm{CaOxSS}$ or CaPhosSS. False negatives (FNs) were defined as patients who had normal 24hour urine levels but had elevated supersaturation of the relevant substance (either $\mathrm{CaOx}$ or $\mathrm{CaPhos}$ ). The sensitivities of 24-hour urine calcium, phosphate and oxalate values for detecting elevated urine supersaturation were determined by the following formula: Sensitivity $=$ TP $/($ TP + FN). These calculations were performed in 2 ways. The first was to determine the sensitivity of each 24-hour urine value individually (calcium, oxalate or phosphate) for detecting elevated $\mathrm{CaOxSS}$ and CaPhosSS. For example, to calculate the sensitivity of 24-hour urine calcium for detecting elevated $\mathrm{CaOxSS}$ according to this method, a positive test was defined as an elevated 24-hour calcium level and a negative test was a normal 24-hour calcium level, without regard to the oxalate level. The second way to calculate sensitivities involved examining both components of a supersaturation level. For CaOxSS, for example, 
patients who had either an elevated 24-hour calcium level or an elevated 24-hour oxalate level had a positive test; a negative test required both calcium and oxalate to be within normal range.

\section{Specificity}

For the specificity analysis, true negatives (TN) were defined as patients who had normal 24-hour urine levels (of calcium, oxalate or phosphate) and who also had normal supersaturation (of $\mathrm{CaOx}$ or CaPhos). False positives (FP) were defined as patients who had elevated 24-hour urine levels but had normal supersaturation. Specificity was calculated by the following formula: Specificity = $\mathrm{TN} /(\mathrm{TN}+\mathrm{FP})$. Specificities were calculated for each 24-hour urine value alone (calcium, oxalate or phosphate) and then for calcium with oxalate and calcium with phosphate as described in the previous section.

Because cut-off values are somewhat arbitrary, we also performed an analysis using a range of different cut-offs to determine the optimum values for maximizing either sensitivity or specificity.

\section{Results}

\section{Patient characteristics}

The age and sex distribution of the patients in this study, as well as treatment regimens at the time of urine collection, are shown in Table 1.

Among the patients, $70 \%$ of men and $50 \%$ of women were on some kind of medical regimen at the time of urine analysis. The percentage of both men and women who had undergone surgical treatment for calculus disease was just under $50 \%$. Twenty-four hour urine chemistry, supersaturation, $\mathrm{pH}$ and volume are shown in Table 2. In general, men had higher 24-hour values of calcium, oxalate and phosphate and higher 24-hour urine volume. Women had higher CaOxSS and CaPhosSS levels.

\section{Sensitivity}

The sensitivity of a 24-hour calcium level for detecting elevated $\mathrm{CaOxSS}$ was $71 \%$ and $79 \%$ for men and women, respectively; for oxalate, the sensitivity was $59 \%$ and $36 \%$ for men and women, respectively (Table 3 ). The sensitivity of having either an elevated 24-hour calcium or oxalate level for detecting elevated $\mathrm{CaOxSS}$ was $88 \%$ and $93 \%$ for men and women, respectively. The sensitivity of 24-hour calcium for elevated CaPhosSS was $74 \%$ and $88 \%$ for men and women, respectively; for phosphate the sensitivity was $57 \%$ and $8 \%$ in men and women, respectively (Table 3 ). The sensitivity of having either an elevated 24-hour calcium or phosphate level for detecting elevated CaPhosSS was $87 \%$ and $88 \%$ for men and women, respectively. The sensitivity of an elevated 24-hour urine calcium level for detecting either an elevated $\mathrm{CaOxSS}$ or $\mathrm{CaPhosSS}$ was $71 \%$ and $79 \%$ in men and women, respectively (data not shown).

\section{Specificity}

An elevated 24-hour calcium level had a specificity

Table 1. Patient characteristics and treatment types

\begin{tabular}{lccc}
\hline & \multicolumn{3}{c}{ No. (and \%)* of patients } \\
\cline { 2 - 4 } Variable & Men, $n=84(56)$ & Women, $n=66(44)$ & Total \\
\hline Mean age, yr, (and SD) & $48(13)$ & $41(13)$ & $92(61)$ \\
All medical treatment & $59(70)$ & $33(50)$ & $35(25)$ \\
$\quad$ Diet & $23(27)$ & $14(21)$ & $55(37)$ \\
Thiazide & $37(44)$ & $18(27)$ & $33(22)$ \\
Citrate & $22(26)$ & $11(17)$ & $20(13)$ \\
Allopurinol & $16(19)$ & $4(6)$ & $7(5)$ \\
Alkalinization & $1(1)$ & $6(9)$ & $4(3)$ \\
Magnesium oxide & $2(2)$ & $2(3)$ & $71(47)$ \\
All surgical treatment & $40(48)$ & $31(46)$ & \\
\hline SD $=$ standard deviation. & & & \\
*Unless otherwise indicated. & & & \\
\hline
\end{tabular}


of $55 \%$ and $48 \%$ for detecting elevated $\mathrm{CaOxSS}$ in men and women, respectively; oxalate had a specificity of $42 \%$ and $79 \%$ in men and women, respectively (Table 3 ). The specificity of having either an elevated calcium or oxalate level for detecting elevated $\mathrm{CaOxSS}$ was $21 \%$ and $38 \%$ in men and women, respectively. An elevated 24hour calcium urine level had a specificity of $60 \%$ for detecting elevated CaPhosSS in both men and women; the specificity of phosphate was $54 \%$ and $88 \%$ in men and women, respectively. The specificity of an elevated calcium or phosphate for detecting elevated CaPhosSS was $41 \%$ and $60 \%$ in men and women, respectively. Finally, the specificity of calcium for detecting either elevated $\mathrm{CaOxSS}$ or CaPhosSS was $62 \%$ and $48 \%$ in men and women, respectively (data not shown).

\section{Sensitivity and specificity with varying cut-offs}

The effect of changing normal calcium level cutoffs on detecting $\mathrm{CaOxSS}$ and CaPhosSS is shown in Figure 1 and Figure 2. For men, the cut-off that maximized both sensitivity and specificity of $\mathrm{CaOxSS}$ detection was $280 \mathrm{mg}$, and for women, the value was about $230 \mathrm{mg}$. For CaPhosSS, the identical results $(280 \mathrm{mg}$ and $230 \mathrm{mg}$, respectively) were seen in men and in women.

\section{Costs}

Performing a true cost comparison is difficult because many of the assays are unavailable to most laboratories and require sending a specimen to a referral laboratory. Additionally, it should be noted that charges are not the same as costs; laboratories are paid a fraction of their retail charges. However, the listed price for ordering a supersaturation test is $\$ 434$ (Litholink Corporation Price List, January 2007). This includes CaOx, CaPhos and uric acid supersaturation as well as a broad range of 24-hour urine levels relevant to stone formation. The cost of performing the equivalent tests in-house (which will not include the

\begin{tabular}{|c|c|c|c|}
\hline Component & Men & Women & Normal levels \\
\hline Calcium & $278(147)$ & $237(130)$ & $\begin{array}{c}\text { Men }<250 \\
\text { women }<200\end{array}$ \\
\hline Oxalate & $47(21)$ & $34(15)$ & Men and women $\leq 40$ \\
\hline Phosphate & $1280(537)$ & $841(340)$ & Men and women $\leq 1200$ \\
\hline $\mathrm{CaO} \times \mathrm{SS}$ & $6.4(3.2)$ & $6.6(3.4)$ & $<8.88$ \\
\hline CaPhSS & $1.3(1.2)$ & $1.7(1.2)$ & $\leq 2$ \\
\hline $\mathrm{pH}$ & $6.08(0.58)$ & $6.31(0.63)$ & \\
\hline Urine volume & $2445(1022)$ & 1894 (838) & \\
\hline
\end{tabular}

\begin{tabular}{|c|c|c|c|c|}
\hline \multirow{2}{*}{$\begin{array}{l}\text { Elevated 24-hour urine } \\
\text { level }\end{array}$} & \multicolumn{2}{|c|}{ Sensitivity, \% } & \multicolumn{2}{|c|}{ Specificity, \% } \\
\hline & Men & Women & Men & Women \\
\hline \multicolumn{5}{|l|}{$\mathrm{CaOxSS}$} \\
\hline Calcium & 71 & 79 & 55 & 48 \\
\hline Oxalate & 59 & 36 & 42 & 79 \\
\hline Calcium or oxalate & 88 & 93 & 21 & 38 \\
\hline \multicolumn{5}{|l|}{ CaPhosSS } \\
\hline Calcium & 74 & 88 & 60 & 60 \\
\hline Phosphate & 57 & 8 & 54 & 88 \\
\hline $\begin{array}{l}\text { Calcium or } \\
\text { phosphate }\end{array}$ & 87 & 88 & 41 & 60 \\
\hline
\end{tabular}


calculated supersaturation levels) will vary from one laboratory to another; however, it should be less expensive than sending the sample to another laboratory.

\section{Interpretation}

The patients in this study have characteristics consistent with serious metabolic stone disease. Most patients were receiving either dietary or medical treatment for metabolic stone disease at the time of urine analysis. One-half of the patients had previously undergone at least 1 surgical intervention for stone disease (and many had undergone several). In this population, a 24-hour urine analysis is often used to follow response to a treatment so that modifications can be made to the treatment regimen. A patient may undergo more than 1 treatment at a given time, and each treatment can have a different effect on the relation between the total amount of a substance in the urine and the supersaturation. For example, a patient may be on a regimen that increases urine volume as well as urine $\mathrm{pH}$. Each of these affects the supersaturation of $\mathrm{CaOx}$ but may not change the total amount of calcium or oxalate excreted in the urine. The distinction is important because supersaturation is

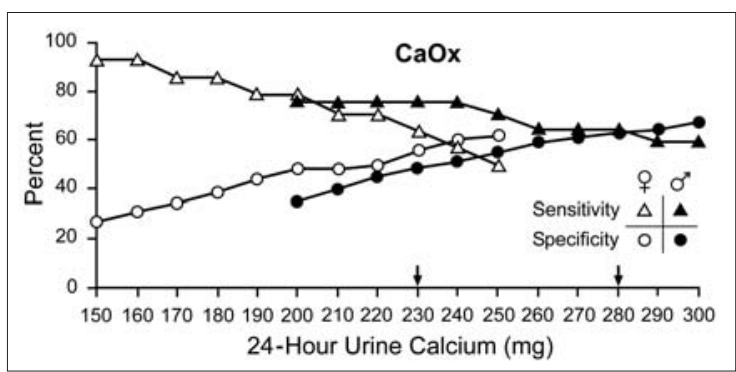

Fig. 1. Sensitivity and specificity of calcium for elevated $\mathrm{CaOx}$ supersaturation. $\mathrm{CaOx}=$ calcium oxalate.

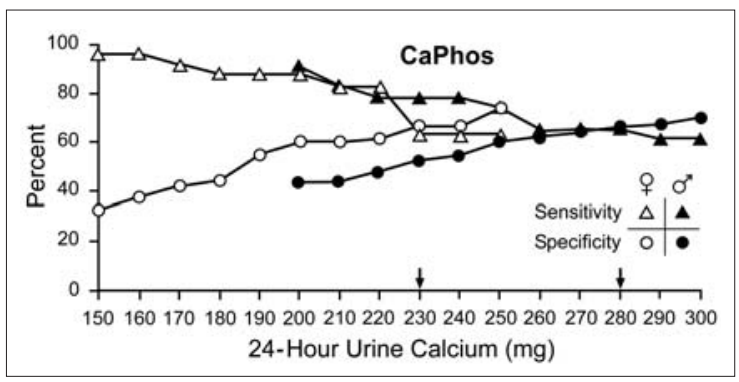

Fig. 2. Sensitivity and specificity of calcium for elevated CaPhos supersaturation. $\mathrm{CaPhos}=$ calcium phosphate. accepted as "the most direct available expression of crystallization potential that one can obtain in clinical practice. ${ }^{\prime 8}$

The sensitivity and specificity of 24-hour calcium for detecting elevated $\mathrm{CaOxSS}$ and CaPhosSS were similar for men and for women. This held true for calcium alone or in combination with oxalate or phosphate. When a positive test was defined as elevation of either component of a substance (i.e., calcium or phosphate elevated for CaPhosSS), the sensitivity was near $90 \%$ for detecting elevated $\mathrm{CaOxSS}$ and $\mathrm{CaPhosSS}$ in both men and women. The specificities of these tests were low, however. With the same definition of a positive test, the specificity of 24-hour calcium and oxalate for $\mathrm{CaOxSS}$ in women was $38 \%$ and in men was $21 \%$. Therefore, most patients with elevated supersaturation of either $\mathrm{CaOx}$ or $\mathrm{CaPhos}$ would be detected by the traditional 24-hour urine analysis. However, many patients with abnormal 24-hour urine analysis will actually have normal levels of CaOxSS and CaPhosSS. The cut-offs for normal 24-hour urine values are arbitrary, and changing them will affect the apparent sensitivity and specificity of these tests. However, even when calculations were repeated with a range of cut-off values, the sensitivity/specificity profile of these tests for detecting elevated supersaturation was poor.

If treatment decisions are based on traditional 24-hour urine analysis results, many patients with normal supersaturation levels will be treated. Because the thermodynamic driving force of stone formation is supersaturation, using only traditional urine analysis for treatment decisions would lead to overtreatment of most patients. Therefore, identification of patients who are truly at increased risk of further stone formation would optimize resource use and minimize patient risk.

These results cannot be generalized to everyone, however, owing to the limitations of this study. Because all patients in this study were known stone formers and most were receiving some kind of treatment at the time of urine analysis, these results may differ from those of treatmentnaive patients undergoing initial metabolic evaluation. Another limitation is that, although supersaturation is thermodynamically a more important factor for stone formation than total urine levels, it is not clear whether patients experience reduced recurrence rates when treatments 
are guided by supersaturation levels instead of total urine levels. Finally, the costs of performing or sending out these tests will vary from one laboratory to another, so a detailed cost comparison from our institution may not be applicable to others. Such a comparison would also overlook the costs (financial and otherwise) of under- or overtreating patients with stone disease as well as the costs of ordering repeat urine studies in patients whose 24 -hour urine values are abnormal.

\section{Conclusion}

In a typical stone clinic population, traditional 24hour urine chemistry analysis is sensitive for detecting elevated $\mathrm{CaOxSS}$ and $\mathrm{CaPhosSS}$, but it lacks specificity. Treatment decisions based on traditional 24-hour urine chemistry analysis would therefore lead to overtreatment in a majority of patients according to the concept of reducing urine supersaturation during nephrolithiasis treatment. Although determining supersaturation requires a send-out test that costs more than traditional urine chemistry analysis, this cost should be balanced against the increased costs and risks of overtreating patients who have normal supersaturation levels.
From the *Department of Urology and the †Division of Nephrology, University of Rochester Medical Center, Rochester, NY

This article has been peer reviewed.

Competing interests: None declared.

\section{References}

1. Finlayson B. Physicochemical aspects of urolithiasis. Kidney Int 1978;13:344-60.

2. Carvalho M, Vieira MA. Changes in calcium oxalate crystal morphology as a function of supersaturation. Int Braz J Urol 2004;30:205-8.

3. Parks JH, Coward M, Coe FL. Correspondence between stone composition and urine supersaturation in nephrolithiasis. Kidney Int 1997;51:894-900.

4. Asplin J, Parks J, Lingeman J, et al. Supersaturation and stone composition in a network of dispersed treatment sites. J Urol 1998;159:1821-5.

5. Coe FL, Wise H, Parks JH, et al. Proportional reduction of urine supersaturation during nephrolithiasis treatment. J Urol 2001;166:1247-51.

6. Siener R, Glatz S, Nicolay C, et al. Prospective study on the efficacy of a selective treatment and risk factors for relapse in recurrent calcium oxalate stone patients. Eur Urol 2003:44:467-74

7. Lingeman J, Kahnoski R, Mardis H, et al. Divergence between stone composition and urine supersaturation: clinical and laboratory implications. J Urol 1999;161:1077-81.

8. Lingeman J, Mardis H, Goldfarb DS, et al. Medical reduction of stone risk in a network of treatment centers compared to a research clinic. J Urol 1998;160:1629-34.

Correspondence: Dr. Erdal Erturk, University of Rochester Medical Center, Department of Urology, 601 Elmwood Ave., Box 656, Rochester NY 14642; erdal_erturk@urmc.rochester.edu

\section{Reprints}

\section{Bulk reprints of $C U A J$ articles are available in minimum quantities of $\mathbf{5 0}$}

For information or orders:

Reprint Coordinator

journal@cua.org

\section{Tirés à part}
On peut obtenir en lots des tirés à part des articles du CUAJ (minimum de 50)

\author{
Renseignements ou commandes : \\ Coordonnatrice des tirés à part \\ journal@cua.org
}

